\title{
Two-Photon Intravital Multicolor Imaging Combined with Inducible Gene Expression to Distinguish Metastatic Behavior of Breast Cancer Cells In Vivo
}

\author{
Sylvia E. Le Dévédec, Reshma Lalai, Chantal Pont, Hans de Bont, Bob van de Water
}

Division of Toxicology, Leiden Amsterdam Center for Drug Research (LACDR), Leiden University, Leiden, The Netherlands

\begin{abstract}
Purpose: The aim of this study is to use multicolor intravital imaging together with an inducible cell model to compare metastatic behavior of control and genetically modified breast cancer cell populations within the intact primary tumor of a mouse.

Procedure: GFP-MTLn3-ErbB1 cells were generated with doxycycline-regulated conditional transgene expression using lentiviral TREAutoR3-cyan fluorescent protein (CFP). CFP expression together with tumor cell motility is monitored in vitro and in vivo.

Results: Effective and tight control of doxycycline-induced CFP expression was observed both in vitro and in vivo. Intravital multiphoton microscopy on intact orthotopic tumors allowed a clear discrimination between GFP-only and (GFP+CFP) cell populations, which enables direct comparison of the motility behavior of two different cell populations in the same microenvironment in vivo. Conclusions: This system is robust and versatile for conditional gene expression and can be used to study the role of individual candidate metastasis genes in vitro and in vivo. This technology will allow investigations of cellular events in cancer metastasis and in particular intravasation within a primary tumor.
\end{abstract}

Key words: Intravital imaging, Two-photon microscopy, Lentiviral inducible system, Metastasis, Breast cancer

Abbreviations RAG mice, Recombinase activating gene; yc, Common gamma chain; FACS, Fluorescence-activated cell sorter; EGFR, Epidermal growth factor receptor; CFP, Cyan fluorescent protein; EGFP, Enhanced green fluorescent protein; TRE, Tetracycline-responsive promoter; RtTA, Reverse tetracycline-responsive transactivator; Doxy, Doxycycline; ECM, Extracellular matrix; TPLSM, Two-photon laser scanning microscopy

\begin{abstract}
Significance: The underlying mechanisms leading to breast cancer metastasis formation are still poorly understood. To enable the analysis of the role of individual candidate metastasis-modifying genes, in this manuscript, we have carefully characterized and determined the feasibility of a methodology that combines the use of an inducible lentiviral expression systems and two-photon intravital multicolor imaging. We anticipate our methodology will have an important impact on the future detailed analysis of tumor cell autonomous mechanisms of metastasis formation, an important area of cancer research.
\end{abstract}

Electronic supplementary material The online version of this article (doi:10.1007/s11307-010-0307-z) contains supplementary material, which is available to authorized users.

Correspondence to: Bob van de Water; e-mail: water_b@lacdr.leidenuniv.nl

\section{Introduction}

$\mathrm{D}$ espite many years of laboratory and clinical research, still a large number of women die of breast cancer metastasis formation [1]. Therefore, it is crucial to understand the underlying mechanisms of metastasis formation and especially tumor cell intravasation [2, 3]. Improved models are required to study the role of individual candidate metastasis genes involved in this individual steps and in particular in the intravasation steps [4].

So far, constitutive ectopic expression of wild-type genes, their deletion mutants, or shRNA-mediated knockdown is 
typically used to study the role of candidate metastasis genes. Often, this by itself affects already the tumor formation and/or is toxic to cells. Indeed, overexpression of a deletion mutant or knockdown of a protein may affect cell proliferation or survival and consequently the tumor growth in vivo, so no conclusion can be drawn on the effect of the candidate gene on tumor cell invasion capacity. In that case, the choice for an experimental metastasis assay is made to still assess the metastatic capacity of the cells. To circumvent this problem, conditional gene expression strategies allowing the expression of target gene only when desired are invaluable tools for cancer research $[5,6]$. For example, after orthotopic injection of the transgenic cell line, ideally, one waits until the tumor reaches a certain size and at a desired moment induces overexpression of a deletion mutant and studies its affect by quantifying the lung burden. An inducible system is relevant not only for studying the effect of gene candidates on metastasis formation but also when combined with dual-color imaging for observing at the same time in the same microenvironment the motility behaviors of the control cells and the genetically modified cells.

Currently, available systems often have limited in vivo functionality because of leakiness, insufficient levels of induction, lack of tissue specificity, or complicated designs $[7,8]$. We evaluated the use of a highly versatile lentiviral vector-based tool that allows conditional expression of any candidate metastasis gene of interest [9]. This approach allows one to analyze the behavior of different manipulated cell populations in the same tumor microenvironment by optical imaging. Two-photon intravital imaging of fluorescent protein-expressing tumors enables cell motility to be observed with great detail and at great depth within tissue [10-13]. In addition to detecting tumor cells, two-photon intravital imaging can provide information about cell movement and interactions between cells and the microenvironment $[12,14,15]$. Our ultimate goal is to compare the metastatic behavior (e.g., number of tumor cells in blood and number and size of lung metastases) of two distinct tumor cell populations (control cells and modified cells with overexpression or knockdown of a target gene) within the same primary tumor. Since there is a correlation between patterns in gene expression in cells with varying metastatic potential and differences in cell motility and polarization in vivo, our aim is to relate the motility cell behavior of the two cell subpopulations and their metastatic capacity. For this purpose, we use two-photon intravital microscopy combined with fluorescently labeled cells. The use of green fluorescent protein (GFP) and cyan fluorescent protein (CFP) can be simultaneously imaged in a living tumor $[12,16]$. In this proof-of-concept study, we chose this fluorophore combination to be able to discriminate between control GFP-labeled cells and GFP-labeled cells that also harbor conditional expression of CFP alone in the same tumor microenvironment.

Here, as a concept, we generated a GFP-MTLn3-ErbB1 cell line that harbors the lentiviral TREAutoR3-CFP con- struct and established the functionality of this lentiviral vector-based tool that allows conditional expression for both in vitro and in vivo study. We systematically characterized the induction kinetics of CFP expression and demonstrated that doxycycline-induced CFP expression does not influence cell proliferation and motility properties in vitro or in vivo. Our data clearly show that two-photon intravital imaging can be used to simultaneously image cell migration of GFP-only and CFP-labeled tumor cells, collagen fibers, and macrophages in established tumors in the living animal. Thus, in the very same tumor microenvironment, we can study the effect of the expression of a gene candidate on the metastatic behavior of individual tumor cells since both control and modified cells can be tracked simultaneously by using twophoton intravital imaging. In the context of metastasis formation, this model will allow the future evaluation of candidate metastasis target genes on tumor cell dissemination programs to the lung.

\section{Materials and Methods}

\section{Reagents}

Mouse antihuman ErbB1 was purchased from Calbiochem (EMD Biosciences, San Diego, CA, USA). Goat antimouse APC was purchased from Cedarlane (Ontario, CA, USA). Alpha modified minimal essential medium ( $\alpha \mathrm{MEM})$, fetal bovine serum, phosphatebuffered saline (PBS), and trypsin were from Life Technologies (Rockville, MD, USA).

\section{Cloning and Lentivirus Production}

The lentiviral vector TREAutoR3 was kindly provided by Dr. Jurgen Seppen [9]. An AgeI-PstI digest of TREAutoR3 released the d2eGFP fragment. The CFP fragment from Clontech vector CFP-C1 was cloned into the AgeI-PstI digest of TREAutoR3 to create the TREAutoR3-CFP vector. Lentiviral vectors were prepared as reported previously [17].

\section{Transfection and Stable Cell Line Selection}

MTLn3 rat mammary adenocarcinoma cells [18] were cultured as previously described [19]. MTLn3-GFP-ErbB1 cell lines were kindly provided by Dr. Jeffrey E. Segall [20] and were maintained in $\alpha \mathrm{MEM}$ (Life Technologies, Inc., Gaithersburg, MD, USA) supplemented with $5 \%$ fetal bovine serum (Life Technologies). TREautoR3 virus containing supernatant was added to the medium of the MTLn3 cells. After 24-h transduction, the medium was refreshed, and after $48 \mathrm{~h}$ the cells were cultured to be frozen at $-80^{\circ} \mathrm{C}$ and selected for CFP expression after doxycycline exposure.

\section{Immunofluorescence}

Cells were cultured on 12-mm collagen-coated glass coverslips and fixated in $4 \%(w / v)$ formaldehyde in PBS. Subsequently, coverslips were mounted in Aqua PolyMount (Polysciences, Warrington, PA, USA). Imaging occurred using a Bio-Rad Radiance 2100 MP 
confocal laser scanning system with a Nikon Eclipse TE2000-U inverted fluorescence microscope and a $\times 60$ Nikon objective.

\section{Cell Proliferation and Motility Assays}

GFP-MTLn3-ErbB1-TREAutoR3-CFP cells were cultured in 24well plates in the absence or presence of doxycycline for 5 days. Cells were washed with PBS and subsequently scanned with a Typhoon Imager 9400 (Amersham Biosciences) with both 457 and $488 \mathrm{~nm}$ laser lines. Total GFP fluorescence which is equivalent with total number of cells was determined with ImageQuant 5.2 software. For cell motility assays, cells were plated onto collagencoated, 24-well, glass-bottom plates (Sensoplate, Greiner Bio-One) and after $2 \mathrm{~h}$ of serum starvation stimulated with either epidermal growth factor (EGF; $10 \mathrm{mM}$ ) or hepatocyte growth factor (HGF; $10 \mathrm{ng} / \mathrm{ml}$ ) and followed with time lapse microscopy for $2 \mathrm{~h}$. Image acquisition was performed using a Nikon TE2000 combined with a Prior stage and controlled by NIS Element Software. Using GFP and CFP filters for epifluorescence microscopy, time lapse videos were recorded using a charge-coupled device (CCD) camera controlled by NIS Element Software.

\section{In Vivo Tumor Growth and Metastasis Formation} Six-week-old $\operatorname{Rag} 2^{-/-} \gamma \mathrm{c}^{-/-}$mice were obtained from in-house breeding. Animals were housed in individually ventilated cages under sterile conditions containing three mice per cage. Sterilized food and water were provided ad libitum. To measure spontaneous metastasis, tumor cells were grown to $70 \%$ to $85 \%$ confluence before being harvested for cell counting. Cells $\left(5 \times 10^{5}\right)$ were injected into the right thoracic mammary fat pads. The cells were injected in a volume of $100 \mu 1$ of PBS with $\mathrm{Ca}^{2+}(1 \mathrm{mM})$ and $\mathrm{Mg}^{2+}$ (2 $\mathrm{mM})$ through a 25-gauge needle. Tumor growth rate was monitored at weekly intervals after inoculation of tumor cells. Horizontal $(h)$ and vertical $(v)$ diameters were determined, and tumor volume $(V)$ was calculated $\left(V=4 / 3 \pi\left(1 / 2[\sqrt{ }(h \times v)]^{3}\right)\right)$. After 4 weeks, the animals were anesthetized with isoflurane, and the lungs were excised and rinsed in ice-cold PBS after having performed intravital imaging (see below). The right lung was used to count the tumor burden. For rough estimation, the right lungs were imaged with the fluorescent imaging unit IVIS (see below). And for detailed quantification, the flat side of the right lung was analyzed with the immunofluorescence microscope. With a $\times 10$ objective lens (NA 0.25), we screened the flat surface of the lobe and counted the number of GFP- or CFP-positive metastases. Following that step, the right lung was cut into two pieces and fixated in $4 \%$ paraformaldehyde. The left lung was injected with ink solution and thereafter destained in water and fixated in Fekete's $(4.3 \%(v / v)$ acetic acid, $0.35 \%(v / v)$ formaldehyde in $70 \%$ ethanol). Lung tumor burden was also determined by counting the number of surface metastases. The primary tumors and lungs from each mouse were used for histologic analysis. Samples were fixed in formalin and embedded in paraffin, and 5- $\mu \mathrm{m}$ sections were stained with hematoxylin and eosin (H\&E).

\section{Intravital Imaging by Multiphoton Microscopy}

Tumor imaging was done as described previously [10, 12, 16, 20]. Cells $\left(5 \times 10^{5}\right)$ were injected into the mammary fat pads of Rag $2^{---} \mathrm{\gamma c}^{---}$ mice as described above and allowed to grow for 4 weeks. The animal was placed under isoflurane anesthesia, and the tumor was exposed using a simple skin flap surgery, with as little disruption of the surrounding vasculature as possible. Eventually, by injecting $200 \mu \mathrm{l}$ of $20 \mathrm{mg} / \mathrm{ml}$ of Texas Red dextran in PBS $2 \mathrm{~h}$ prior to imaging, we can also visualize the blood vessels and the macrophages in the primary tumor $[12,15]$. The animal was then placed in a $30^{\circ} \mathrm{C}$ temperature chamber on an inverted Nikon TE2000-U microscope and imaged using a $\times 20$ Nikon objective (Plan Apo, NA 0.75 ). Briefly a 5-W Tsunami laser (Spectra Physics, Mountain View, CA, USA) was used to run a Radiance 2100 multiphoton system (Bio-Rad, Hercules, CA, USA) at $860 \mathrm{~nm}$ using a 450/480-nm filter to image matrix and CFP and a 515/530-nm filter to image CFP and GFP. Time lapse images of CFP and GFP-labeled MTLn3-ErbB1 generated tumors were taken at 1 -min interval for $30 \mathrm{~min}$. The images were collected using Bio-Rad's Lasersharp 2000 software. During each 1-min interval, a $z$ series of ten images was taken at a spacing of $10 \mu \mathrm{m}$ between images, beginning at the periphery of the tumor and moving into the tumor. For each tumor, this image acquisition process was repeated for $30 \mathrm{~min}$, resulting in a time lapse three-dimensional $z$ series for analysis of tumor cell motility. Image analysis and cell tracking were done with Image Pro Software.

\section{Fluorescent Imaging}

Fluorescent imaging was performed with a highly sensitive, cooled CCD camera mounted in a light-tight specimen box (IVIS ${ }^{\text {TM}}$; Xenogen). Imaging was controlled by the acquisition and analysis software Living Image ${ }^{\circledR}$ (Xenogen). The light emitted from the biofluorescent metastases or cells were detected by the IVIS ${ }^{\text {TM }}$ camera system, integrated, digitized, and displayed. For ex vivo imaging, lungs were excised, placed into a petri dish, and imaged for $1-2 \mathrm{~min}$.

\section{Statistical Analysis}

Student's $t$ test was used to determine if there was a significant difference between two means $(p<0.05)$. When multiple means were compared, significance was determined by one-way analysis of variance $(p<0.05)$. Significant differences are marked in the graphs.

\section{Results}

\section{Conditional Transgene Expression In Vitro}

To study two different cell populations (with differential transgene expression) in the same microenvironment in vivo, a model is required that uses two different fluorescent proteins in order to discriminate these populations. Moreover, given the possible effect of transgene expression or knockdown on tumor growth and the microenvironment, such a transgene expression should be conditional. Here, we chose to work with GFP and CFP, two fluorescent proteins that can be visualized by two-photon intravital imaging and thus in vivo [16]. Furthermore, to test whether a cell line with inducible expression of candidate metastasis controlling genes can be used for in vivo studies, we used the established GFP-MTLn3-ErbB1 cell line and verified first the functionality of the lentiviral vector TREAutoR3 in vitro $[9,20,21]$. We generated a TREAutoR3 lentiviral vector 
that expresses CFP after doxycycline-mediated induction to create breast cancer cells that either lack or express a transgene upon treatment with doxycycline (Fig. 1a). We transduced GFP-MTLn3-ErbB1 cells, and directly after transduction, we added doxycycline to sort the positive cells by FACS analysis for both CFP and GFP expressions (data not shown). In addition, similar levels of ErbB1 were observed in both the original cell line (GFP-MTLn3-ErbB1) and the new cell line (GFP-MTLn3-ErbB1-TREAutoR3-CFP) (data not shown). Next, we determined whether the expression of the transgene was tightly and effectively controlled by doxycycline. CFP expression was upregulated after $24 \mathrm{~h}$ of doxycycline treatment with all concentrations. The most optimal concentration was $1,000 \mathrm{ng} / \mathrm{ml}$ at which over $90 \%$ of the cells were CFP positive (Fig. 1b). In the absence of doxycycline, no CFP expression was observed, indicating that transgene expression was tightly and effectively controlled by doxycycline (Fig. 1b). We also checked the kinetics of the CFP transgene expression over time. After $8 \mathrm{~h}$, around $50 \%$ of the cells were CFP positive, while after $24 \mathrm{~h}$ already approximately $90 \%$ of the cells were CFP positive (Fig. 1c). Together, these data show that the autoregulatory lentiviral vector TREautoR3 does not leak at all
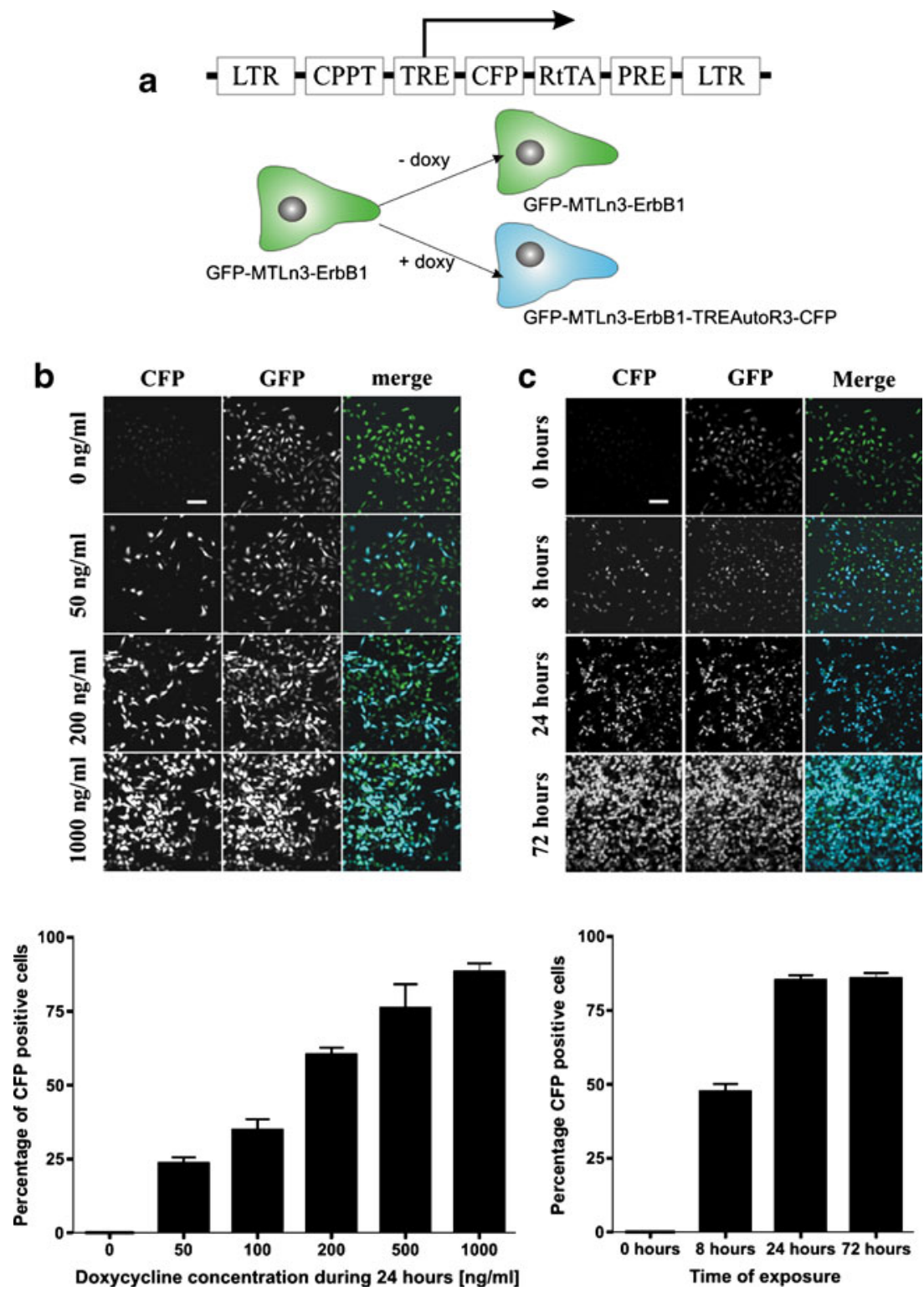

Fig. 1. In vitro drug-controllable transgene expression. a Schematic representation of the constructed lentiviral vector. b Doxycycline dose-response of the TRE-autoR3-CFP vector. Transduced GFP-MTLn3-ErbB1 cells were cultured in the absence or presence of doxycycline at different concentrations for $24 \mathrm{~h}$ (from 50 until 1,000 $\mathrm{ng} / \mathrm{ml}$ doxy) and visualized by confocal microscope. In this titration experiment, the percentage of CFP-positive cells was calculated. c Induction kinetics of the CFP expression of GFP-MTLn3 cells transduced with TREautoR3 vector. Dual-color imaging of cells exposed to 1,000 ng/ $\mathrm{ml}$ of doxycycline after different time points. The number of CFP-expressing cells after doxycycline induction was counted. Scale bar for $\mathbf{b}, \mathbf{c}=100 \mu \mathrm{m}$. 
in the absence of the drug and that the regulation of the transgene expression is sensitive, fast, and tight. Moreover, with this one cell line with inducible expression of CFP, we generated a versatile tool to study the effect of transgene expression compared to control situation in one experiment where all conditions are exactly the same.

\section{Doxycycline-Mediated Expression of CFP in MTLn3 Cells Does Not Affect Cell Proliferation and Cell Motility}

The use of such an inducible system together with CFP expression might affect cell properties. Therefore, we carefully checked whether doxycycline-mediated expression of CFP alone affects two important aspects in the breast cancer progression: cell proliferation and cell motility. To determine the effect of doxycycline-mediated CFP expression on cell proliferation, our conditional cell line was cultured in 24 wells for 5 days either untreated or treated with doxycycline to induce CFP expression. At indicated time points, the increase in GFP and CFP fluorescence was determined as a measurement of proliferation using a fluorescent imager. After 5 days, no significant effect was observed in cell proliferation rate when cells were exposed to doxycycline (Fig. 2a). Next, we verified whether cell motility remains the same after doxycycline-induced CFP expression. Cell motility of GFP-only or GFP/CFP cells was determined using a random cell migration assay with wide field fluorescent microscopy. No significant difference was observed in cell motility between GFP and GFP/CFP cells under control conditions or after stimulation of the cells with either EGF or HGF (Fig. 2b). Thus, doxycycline-mediated expression of CFP does not affect in vitro cell proliferation and motility.

\section{Effective Doxycycline-Induced CFP Expression In Vivo Does Not Affect Tumor Growth and Lung Metastasis Formation}

We also checked whether doxycycline-mediated expression of CFP alone affects tumor growth and lung metastasis formation. We used inbred $\mathrm{Rag} 2^{-/-} \gamma \mathrm{c}^{-/-}$mice and injected orthotopically in the fat pad 500,000 GFP-MTLn3-ErbB1TREAutoR3-CFP cells. Three groups were defined based on the doxycycline administration time point. The control group received sucrose drinking water without doxycycline. The second group received doxycycline sucrose drinking water starting from day 1 and the third group starting from day 28 , which is approximately $48 \mathrm{~h}$ before sacrificing the animals (Fig. 3a). We measured the tumor progression with a caliper during the entire length of the experiment, and no significant difference was found in tumor growth rate between the three groups (data not shown). After sacrifice, tumor volume and weight were measured with no differences observed between different treatments (Fig. 3b). Also, histological analysis of tumor tissue sections is similar in the three test groups (Fig. 3c). Next, we quantified with both fluorescence imaging (FLI; Fig. 4a) and fluorescence microscopy a
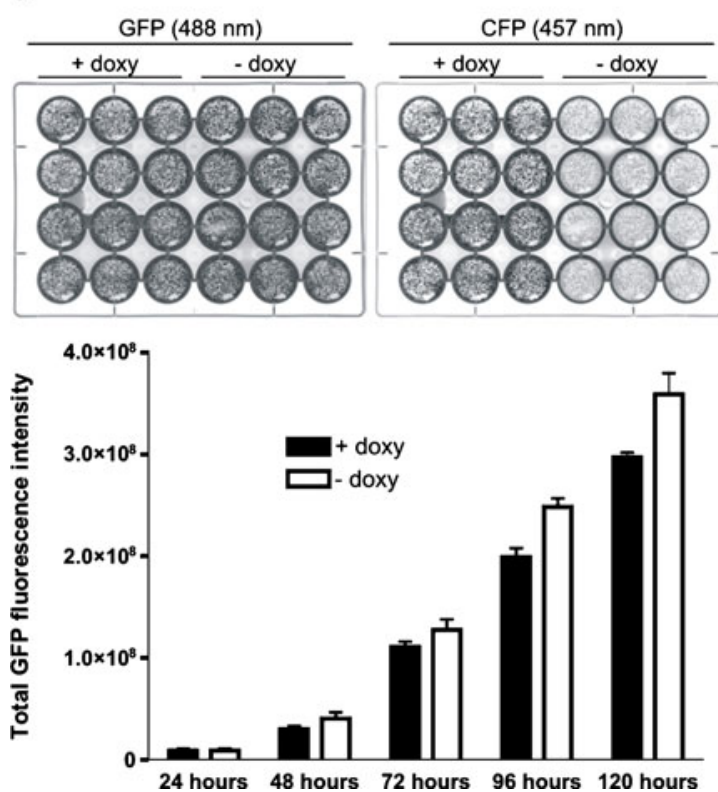

b
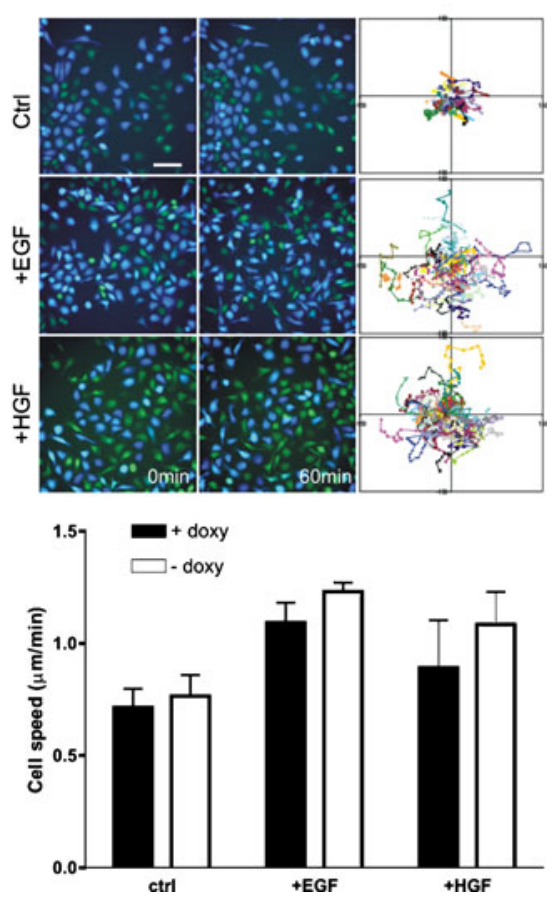

Fig. 2. Doxycycline-mediated CFP expression does not affect cell proliferation and migration in vitro. a Cell proliferation in the absence or presence of doxycycline $(1,000 \mathrm{ng} / \mathrm{ml})$ quantified by measuring the total GFP fluorescence over the time using the Typhoon imager. $\mathbf{b}$ Cell motility determined in a random cell migration assay after EGF or HGF stimulation in the presence or absence of doxycycline. Cells were followed with epifluorescence for $2 \mathrm{~h}$. Scale bar for $\mathbf{b}=50 \mu \mathrm{m}$. 
a

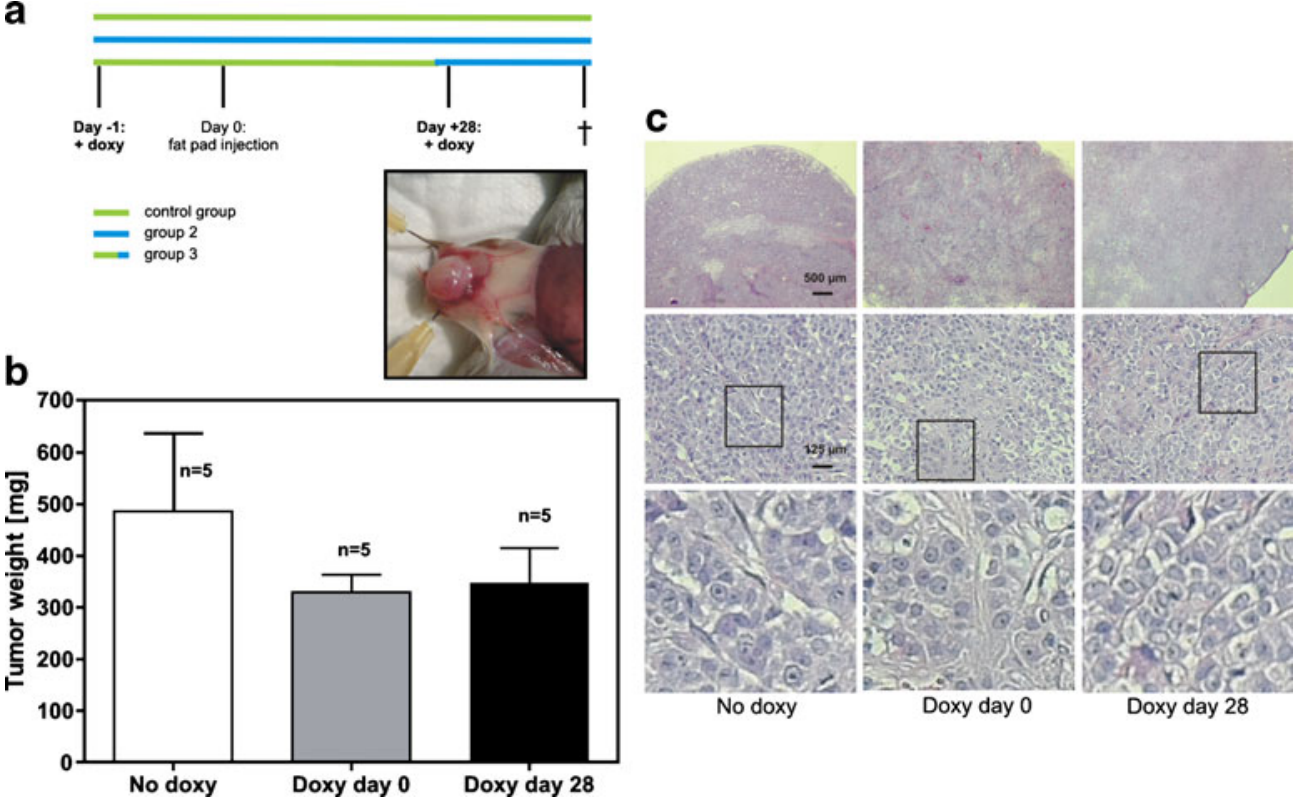

Fig. 3. Doxycycline-mediated CFP expression does not affect tumor cell growth in vivo. a Schedule of in vivo doxycycline treatment. We defined in total three groups: control group $(n=5)$ which gets only sucrose drinking water, group $2(n=5)$ which gets doxycycline in the drinking water from day 0 , and group $3(n=5)$ which gets doxycycline in the drinking water $48 \mathrm{~h}$ before sacrifice. b At sacrifice, the tumor was isolated and the weight measured. c Sectioned tumors were stained with H\&E, and representative pictures are shown.

(Fig. 4b) the lung tumor burden. In all the three groups, an average number of 400 lung metastases were counted, showing no difference in metastasis formation after doxycycline-induced CFP expression. Histological observations of the lungs did not reveal any difference between the three groups (Fig. 4c). In conclusion, tumor growth and lung metastasis formation are not affected by doxycyclineinduced CFP expression.

\section{Tightly Controlled CFP Expression in Both Tumor and Lung Metastases}

Since we want to use this inducible system in vivo, it was essential to evaluate the effectiveness of doxycyclineinduced CFP expression. With two-photon intravital imaging, we checked whether doxycycline CFP induction was successful in the tumor and in the lung metastases. No CFP expression at all was detected in the control group, yet robust CFP expression was detected in over $90 \%$ of the whole tumor when doxycycline was added in the drinking water from day 1 and in more than $70 \%$ of the tumor when doxycycline was added $48 \mathrm{~h}$ before sacrificing the animals (Fig. 5a). We observed the same CFP induction when analyzing the lung metastases with two-photon excitation after sacrifice (Fig. 5b): in the control group, only GFP-positive and no CFP-positive metastasis could be observed throughout the entire lung. When doxycycline was added in the drinking water from day 1 , almost all the lung metastases were CFP positive. A period of 48-h doxycycline exposure resulted in approximately $70 \%$ CFP-positive lung metastasis. Together, these data demonstrate that our GFP-MTLn3-ErbB1-TREAutoR3 model allows for the effective, fast, and tightly controlled transgene expression. Moreover, we can easily discriminate the GFP-only and GFP/ CFP-positive lung metastases which are a prerequisite to evaluate the effect of transgene expression on lung metastasis formation.

\section{Two Distinct Tumor Cell Subpopulations Can Be Tracked in the Same Tumor Microenvironment and Doxycycline-Induced CFP Expression Does Not Affect Cell Motility In Vivo}

Multiphoton imaging technology can be used to get an insight into the microenvironment and understand its role in the invasion and intravasation steps of metastasis inside living mammary tumors $[11,12,14,20]$. Results show that the tumor microenvironment is a dynamic place where interactions between tumor cells, macrophages, blood vessels, and extracellular matrix (ECM) fibers define the metastatic phenotype $[13,15,22]$. In our setup, we used a Bio-Rad Radiance 2100 multiphoton microscope with an inverted Nikon TE2000 connected to a Spectra Physics Tsunami Ti-Sapphire laser of $5 \mathrm{~W}$. As previously shown by E. Sahai and colleagues [16], wavelengths around $880 \mathrm{~nm}$ were most suitable for simultaneous imaging of GFP and CFP. We used for all experiments $860 \mathrm{~nm}$. The selected emission filters enabled us to visualize at the same time CFP (480/30), GFP (515/30), and the extracellular matrix (450/80) simultaneously (Fig. 7 and supplementary movie 1). Collagen type I which is very abundant in the tumor microenvironment can be visualized with second harmonic generation generated by the pulsed laser used in two-photon microscopy [23-25]. In 


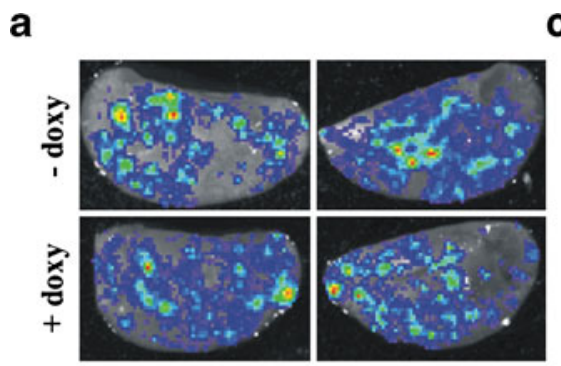

b

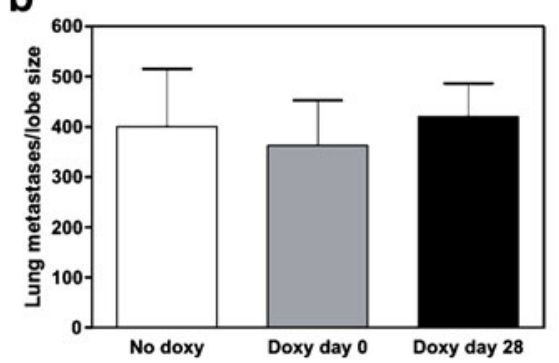

C
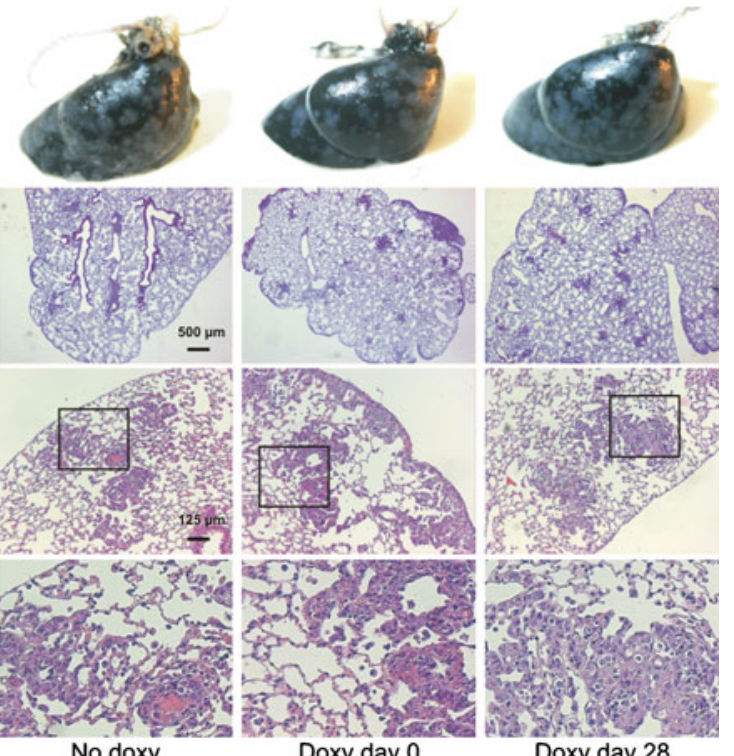

Doxy day 0

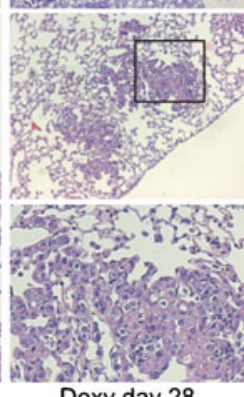

Doxy day 28

Fig. 4. Doxycycline-mediated CFP expression does not affect the number of lung metastases. a Fresh isolated lungs were visualized with FLI. Control group is "- doxy" and group 2 is "+ doxy." b Using epifluorescence, we counted the number of fluorescent lung metastases only on one side of the small lobe. $\mathbf{c}$ Part of the isolated lungs was injected with ink and the rest of the lungs were stained with $\mathrm{H} \& \mathrm{E}$, and representative pictures are shown.

addition, when injecting Texas Red dextran (620/60) in the tail vein of the animal $2 \mathrm{~h}$ prior to imaging, the blood flow and the macrophage in the tumor can be visualized. Together, this provides information about the tumor microenvironment and its host cells (Fig. 6a and supplementary movie 2).

To get a better insight into metastasis mechanisms, we want to be able to track two cell subpopulations within a primary tumor which differentially express a candidate metastasis target gene by using two-photon intravital imaging. For this purpose, we checked firstly whether we were able to discriminate the GFP-only and GFP/CFP cells, and secondly whether the tumor cell motility in vivo was not affected by doxycycline-induced CFP expression. We performed two-photon intravital imaging in primary tumors

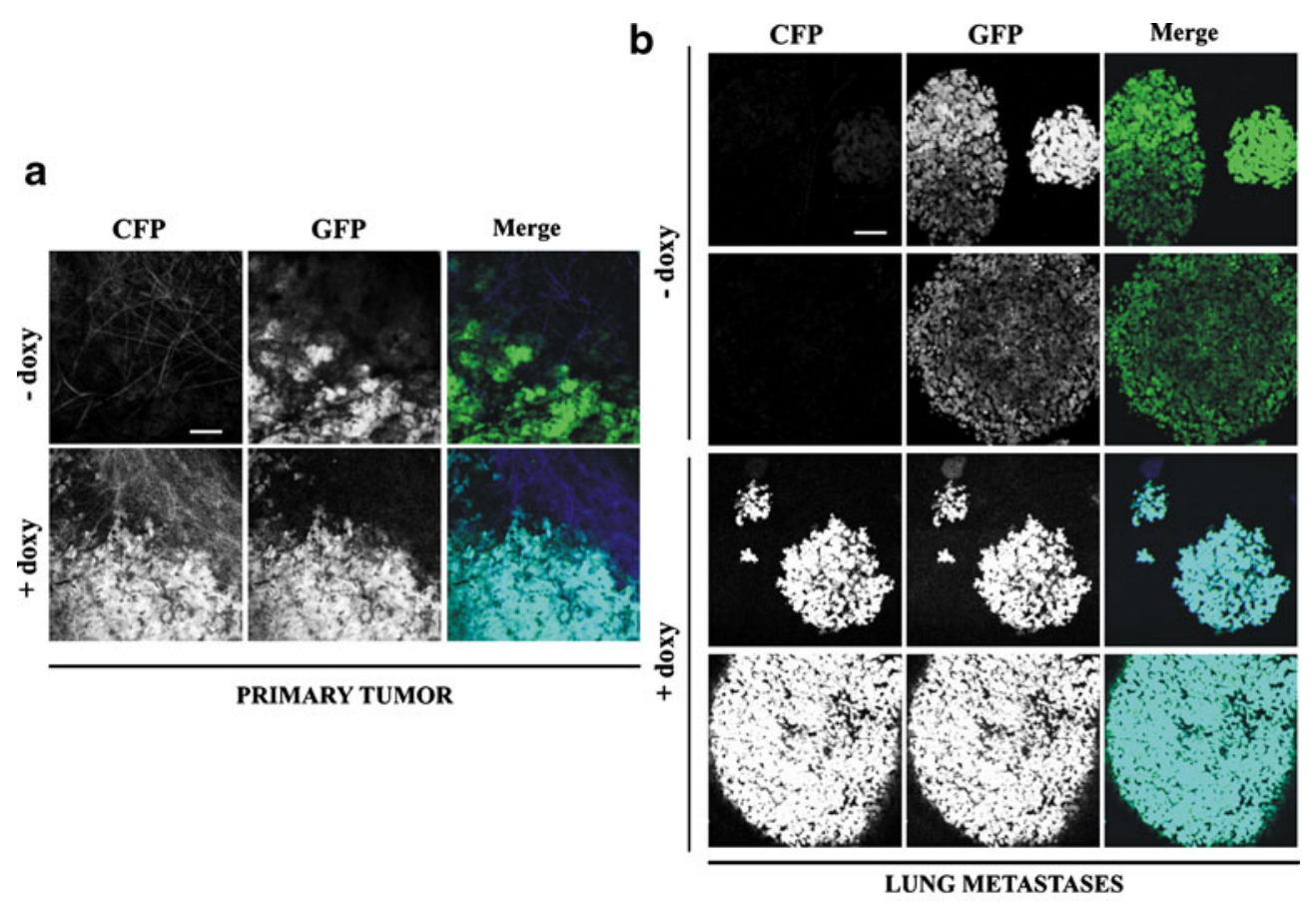

Fig. 5. Tightly controlled CFP expression in both tumor and lung metastases. Fresh isolated lungs and tumors were imaged with multiphoton microscopy. Control group is "- doxy" and group 2 is "+ doxy." Simultaneous capture of GFP, CFP, and collagen second harmonic fluorescence (b/ue) in the mammary tumors (a) and lung metastases (b) of control group and group. Scale bar for $\mathbf{a}, \mathbf{b}=100 \mu \mathrm{m}$. 

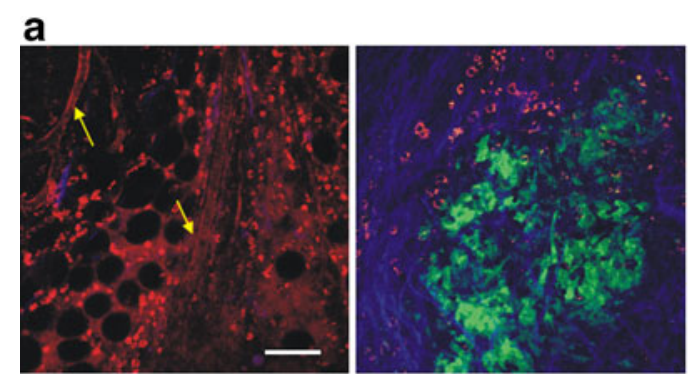

Texas-Red

Texas-Red/GFP/SHG

b

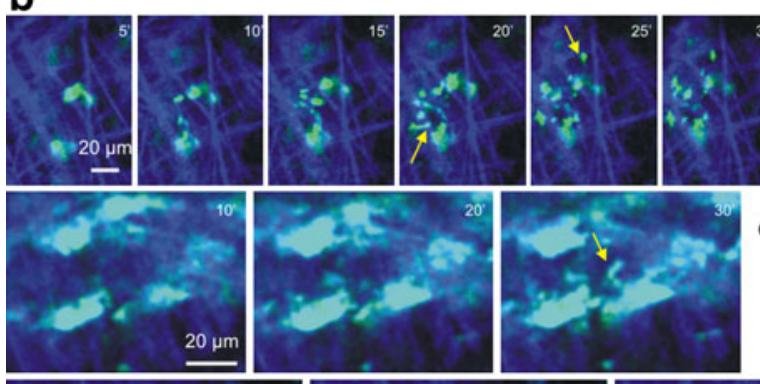

\section{C}
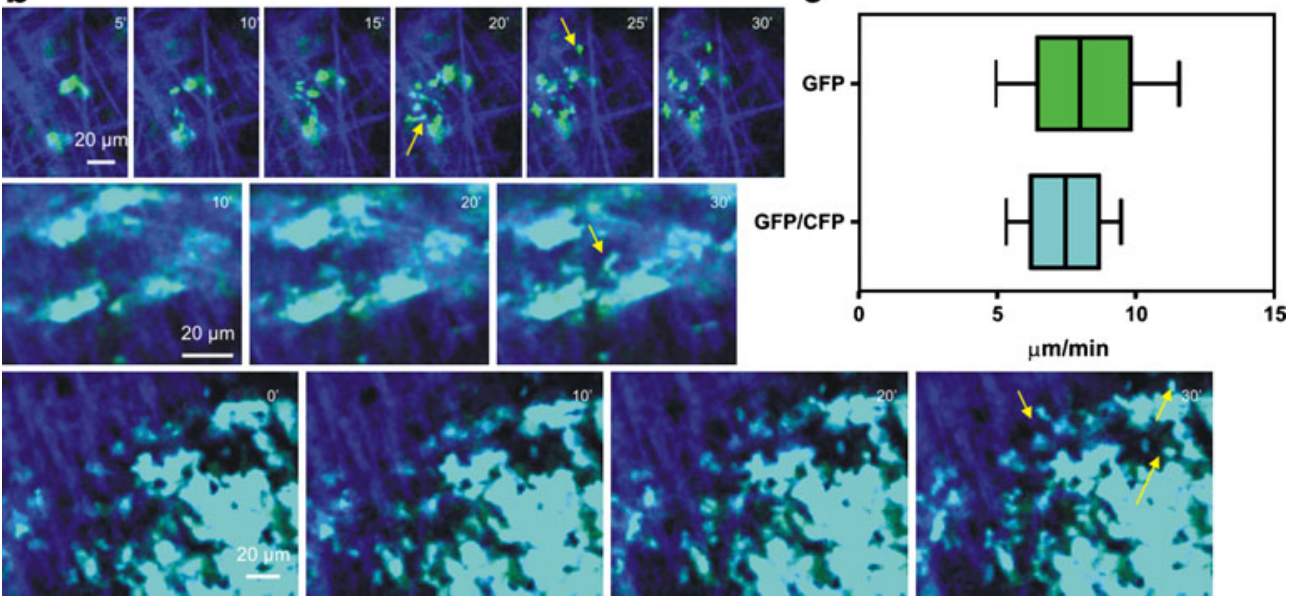

Fig. 6. The microenvironment of a mammary tumor can be imaged using multiphoton microscopy. a Simultaneous imaging of macrophages (red) that are seen outside dextran-labeled vessels (arrow) and the tumor cell mass (green). b Differences in motility can be determined by imaging cells with different transgene expression in the same tumor. The panels show time series of images of mammary tumor with cells either expressing GFP only or combined GFP/CFP after doxycycline induction. c Doxycycline-induced CFP expression does not affect cell motility in vivo. The tumor cells move at a rate of around $7.5 \mu \mathrm{m} / \mathrm{min}$ over the course of the 30-min time lapse. Scale bar for $\mathbf{a}, \mathbf{b}=100 \mu \mathrm{m}$.

of both control and doxycycline groups. We could easily detect, discriminate, and track the two tumor cell subpopulations: GFP-only and GFP/CFP cells (Fig. 6b). We also observed equal amount of cell migration events in both groups. The very few observed migrating cells were solitary with an ameboid morphology. Protrusion, crawling, and host cell events were equally observed in the three animal groups. These cells were often observed moving linearly in association with the ECM fibers. Figure $6 \mathrm{~b}$ (and supplementary movies 3 to 5) shows time series of both GFP-only cells (green) and GFP-CFP (cyan) cells moving along collagen fibers as imaged by second harmonic generation (blue). After tracking the cells with image analysis software, we could determine the cell speed of both cell types. This speed was approximately $7.5 \mu \mathrm{m} / \mathrm{min}$, which is nearly ten times faster as normally observed on two-dimensional substrates in vitro (Fig. 2b). Doxycycline-induced CFP expression did not affect average migration speed of individual tumor cells (Fig. 6c). These data fit with the identical metastatic spread to the lungs under both conditions (Fig. 4a, b). In summary, we can directly compare the behavior of cells with different phenotype that can be tightly controlled by doxycycline induction in the same tumor environment in vivo.

\section{Discussion}

A better understanding of the mechanisms of increased tumor cell motility and migration within the primary breast tumor is required. Constitutive expression of a transgene or knockdown of a gene in breast cancer cells may affect critical processes that are required for primary tumor growth. To study the role of specific candidate metastasis genes on tumor cell motility and subsequent metastasis formation, a model is needed in which gene expression can be induced after a primary tumor is formed. Here, we set up and describe a breast cancer model in which gene expression can be induced at any time point during tumor progression using a doxycycline-dependent conditional gene expression breast cancer cell line combined with two-photon intravital imaging. This model enabled us to study tumor cell motility and intravasation processes of the control and manipulated breast cancer cell populations within the same primary tumor environment.

Currently available drug-inducible systems have often limited in vivo functionality because of leakiness and insufficient levels of induction [7, 8]. Our results demonstrate a high degree of robustness and versatility in allowing drug-controllable transgene expression in comparison to 
classical systems based on transactivation of synthetic promoters [7, 8]. A similar system for conditional transgene expression and gene knockdown has been recently described for its efficiency and versatility but not yet in the dual-color imaging model we are aiming for $[6,26]$. Indeed, Szulc J. and coworkers designed a lentiviral vector-based that controls conditionally the regulation of endogenous proteins in vitro and in vivo. They were able to perform in vivo doxycycline switches to turn endogenous tp53 expression off in a primary tumor at both RNA and protein levels. The GFP marker contained in the same vector was used to track the cells with a knockdown. Since GFP could be replaced by CFP, we also could use this construct to track the cells with a knockdown of a specific metastasis-related protein using two-photon intravital microscopy.

Cancer cell migration in primary tumors can be directly observed by multiphoton microscopy $[11,13]$. Two-photon laser scanning microscopy (TPLSM) offers important advantages over laser scanning microscopy, such as deep tissue penetration and three-dimensionally localized excitation. Here, we used an already well-described pair of fluorescent proteins in dual-color imaging with TPLSM, GFP, and CFP. Nowadays, EGFP and the monomeric version of DsRed (mRFP1) are widely used for dual-color imaging experiments using laser scanning confocal microscopy [27-35]. Nonetheless, this combination of fluorophores is not yet very suitable for twophoton intravital imaging. Indeed, the high-intensity pulsed infrared lasers still commonly used for two-photon microscopy produce light in the 750-980-nm range and are unable to excite RFPs efficiently in deep tissue since the output of most of TiSapphire lasers drops off significantly with the increasing wavelength. Thus, the combination of fluorophores GFP/CFP to track two different subpopulations is still the most suitable for the common two-photon microscope setups. More importantly, with this pair of fluorophores, there is still a free window to use fluorescent molecules in the far red spectrum such as Texas-Red-conjugated dextran to detect blood vessels in the primary tumor which is crucial for visualizing intravasation events. So during image acquisition, we are able to visualize and track tumor cell subpopulations, the blood vessels and macrophages, and the extracellular matrix, all the different components indispensable to understanding mechanisms of metastasis formation. The group of E. Sahai is using this dualcolor imaging to monitor tumor motility of two cell subpopulations in vivo. For instance, using multiphoton confocal imaging in a mixed tumor population, MDA-MB-231 C15-4GFP parental control cells was found to be more motile than the MDA-MB-231 15-20-CFP MRTF-depleted cells [36]. Very recently, they also performed intravital imaging of a "mosaic" tumor containing MTLn3 CFP-expressing control cells and

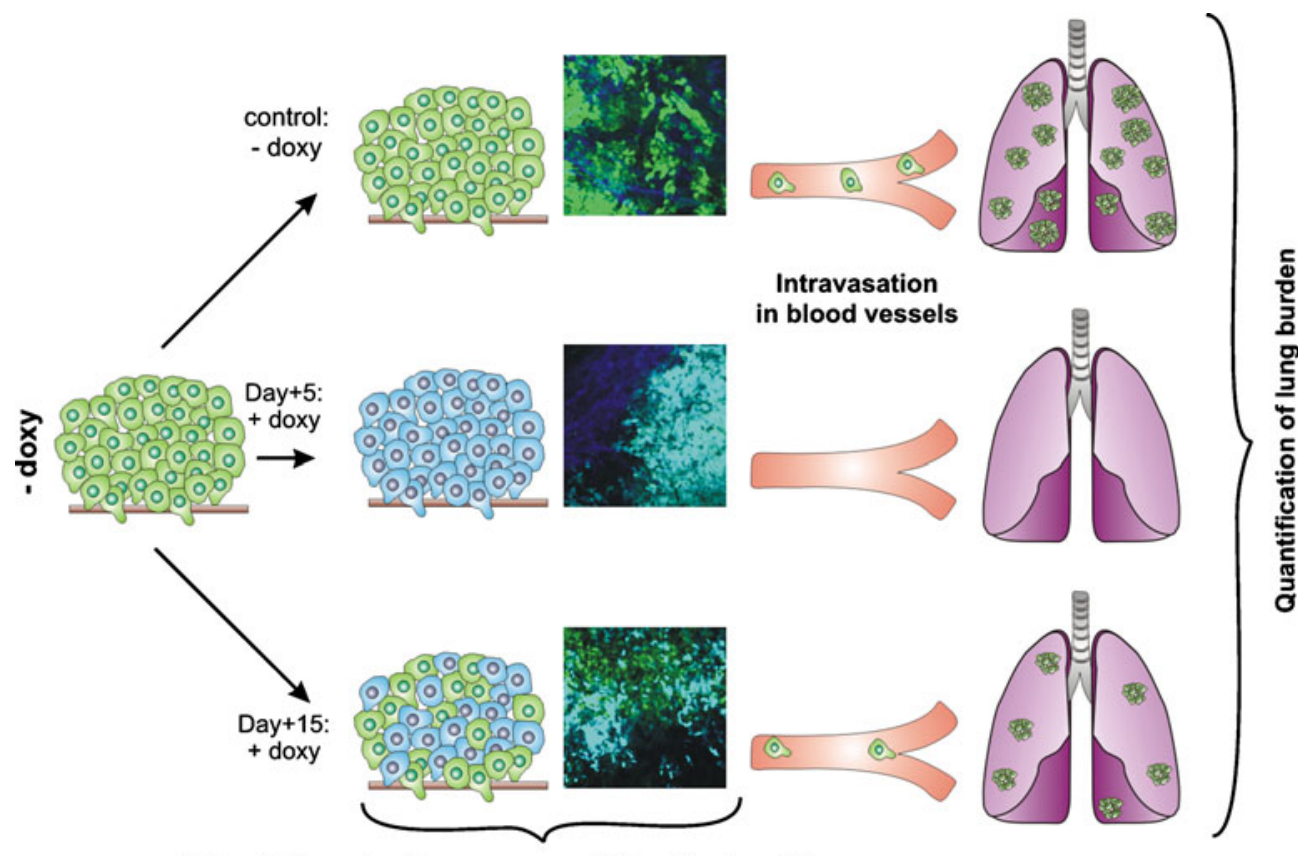

Intravital Imaging to compare motility of both cell types

Fig. 7. Schematic view of the methodology illustrated with an example (conditional expression of a CFP-tagged dominant negative protein that alters cell migration). Control cells are injected in the mammary fat pad and allowed to grow in a primary tumor without doxycycline exposure. In general, three groups of animals can be included in the experimental setup: (1) a control group to assess the metastatic capacity of the control GFP-MTLn3-ErbB1 cells (lungs full of GFP-positive metastases), (2) a series of animals where expression of the CFP-tagged dominant negative protein can be induced at different time points during the experiment (the earlier the induction, the lower the number of GFP-positive lung metastases), (3) a final group to perform intravital imaging in which CFP expression is induced half-way through the experiment (not all the cells are CFP positive so that we can compare the motility behaviors of the control and the modified cells). 
MTLn3 transforming growth factor (TGF)- $\beta$ R-DN-GFPexpressing cells [37]. Analysis of time lapses made in the "mosaic" tumors revealed that the overexpression of a dominant negative of the TGF- $\beta$ receptor completely inhibits single-cell movement while control cells still exhibit both single and cohesive cell migrations. Our approach combines both a relevant inducible breast cancer model together with a successful dual-color imaging. In this way, we can quantify the effect of targeting a certain gene in metastasis formation and correlate those data with the distinct motility behavior analyzed with intravital imaging. In the case of an overexpression of a dominant negative protein (Fig. 7), a potential experimental setup would include (1) a control group which is not exposed to doxycycline and which cells metastasize to the lungs, (2) a series of treatment groups exposed at different times to doxycycline to evaluate the reduction of lung metastases, and (3) a final group exposed only at the end of the experiment to doxycycline so that not all the cells are CFP positive which allow intravital imaging to determine the motility behavior of both cell types and correlate it to their metastatic capacity. We anticipate no problem in discriminating GFP and (GFP + CFP) cells due to the relative expression of the fluorophores since an even low CFP signal can be easily detected.

Our presently described methodology is based on an endpoint observation of the tumor. It would be more efficient to know exactly at what time point the angiogenic switch, thereby allowing and facilitating intravasation of tumor cells, takes place so that the intravital imaging of the primary tumor would reveal many cell events of intravasation. A combination of monitoring the tumor progression with a noninvasive method (whole-body imaging with fluorescence or bioluminescence) and TPLSM where tumors can be visualized at the single-cell resolution would be very powerful, thus predicting the most optimal moment to monitor a maximum number of intravasation events within the primary tumor. Still whole-body imaging using fluorescence is not yet as optimal as bioluminescence although promising results have described the use of a new fluorescent variant tdTomato $[38,39]$. The latter could also be used for TPLSM since its brightness and photostability are much better than mRFP or mCherry. Recently, a new fluorophore pair EGFP-mKeima has been also described as optimal for two-photon dual-color imaging [40]. If mKeima is as performant for whole-body imaging as tdTomato, this new pair EGFP-mKeima could suit both whole-body imaging and TPLSM.

To avoid the end-point experiment due to the skin flap surgery needed to access the primary tumor for visualization, Kedrin and coworkers just published a new technique perfectly adapted for monitoring tumor cell migration within a mammary primary tumor for prolonged time [41]. They design a mammary imaging window so that there is no need any more to perform a skin flap surgery and most of all to sacrifice the mouse after imaging. Moreover, it is now possible to monitor tumor cell migration for more than $24 \mathrm{~h}$ and at different time points during tumorigenesis. A perfect model for understanding metastatic behavior of breast cancer cells would be the use of this new technique combined with our doxycycline-dependent conditional gene expression cell line.

Currently, our established multicolor tumor model system allows visualization of tumor cells with two different phenotypes that may interact with their microenvironment such as the extracellular matrix and other tumor host such as macrophages. Tumors with distinct cell populations and its microenvironment (macrophages and ECM) are uniquely identified by their fluorescence colors. The multicolor tumor cell-environment interaction model system allows observations at the single-cell level in fresh tissue, affording further insights into the role of a specific protein in relation with the tumor microenvironment in tumor growth and progression and especially tumor cell motility. This is particularly very important for understanding the intravasation process. The model will now be used to elucidate the role of target genes within tumor cells with predicted roles in breast cancer progression. Our model is also excellently suited to evaluate the effect of targeted therapeutics that should block cell motility and consequently intravasation and thereby tumor cell dissemination.

Acknowledgments. We thank J. Wyckoff and J. Segall for helpful suggestion for our intravital imaging work and M. de Graauw for valuable discussion and critically reading the manuscript. This work was financially supported by grants from the Dutch Cancer Society (UL 2006-3538 and UL 20073860), the EU FP7 Health Program Metafight (Grant agreement no.201862), the Dutch Organization for Scientific Research (902-21-229 and 911-02-022), and TI Pharma (T3-107).

Open Access. This article is distributed under the terms of the Creative Commons Attribution Noncommercial License which permits any noncommercial use, distribution, and reproduction in any medium, provided the original author(s) and source are credited.

\section{References}

1. Ferlay J, Autier P, Boniol M et al (2007) Estimates of the cancer incidence and mortality in Europe in 2006. Ann Oncol 18:581-592

2. Pantel K, Brakenhoff RH (2004) Dissecting the metastatic cascade. Nat Rev Cancer 4:448-456

3. Gupta GP, Massague J (2006) Cancer metastasis: building a framework. Cell 127:679-695

4. Condeelis J, Singer RH, Segall JE (2005) The great escape: when cancer cells hijack the genes for chemotaxis and motility. Annu Rev Cell Dev Biol 21:695-718

5. van Nimwegen MJ, Verkoeijen S, van Buren L, Burg D, van de Water B (2005) Requirement for focal adhesion kinase in the early phase of mammary adenocarcinoma lung metastasis formation. Cancer Res 65:4698-4706

6. Szulc J, Wiznerowicz M, Sauvain MO, Trono D, Aebischer P (2006) A versatile tool for conditional gene expression and knockdown. Nat Methods 3:109-116

7. Kafri T, van Praag H, Gage FH, Verma IM (2000) Lentiviral vectors: regulated gene expression. Mol Ther 1:516-521

8. Lamartina S, Silvi L, Roscilli G et al (2003) Construction of an rtTA2 (s)-m2/tts(kid)-based transcription regulatory switch that displays no basal activity, good inducibility, and high responsiveness to doxycycline in mice and non-human primates. Mol Ther 7:271-280 
9. Markusic D, Oude-Elferink R, Das AT, Berkhout B, Seppen J (2005) Comparison of single regulated lentiviral vectors with rtTA expression driven by an autoregulatory loop or a constitutive promoter. Nucleic Acids Res 33:e63

10. Condeelis JS, Wyckoff J, Segall JE (2000) Imaging of cancer invasion and metastasis using green fluorescent protein. Eur J Cancer 36:16711680

11. Condeelis J, Segall JE (2003) Intravital imaging of cell movement in tumours. Nat Rev Cancer 3:921-930

12. Kedrin D, Wyckoff J, Sahai E, Condeelis J, Segall JE (2007) Imaging tumor cell movement in vivo. Curr Protoc Cell Biol Chapter 19:Unit 19.7

13. Sahai E (2007) Illuminating the metastatic process. Nat Rev Cancer 7:737-749

14. Sidani M, Wyckoff J, Xue C, Segall JE, Condeelis J (2006) Probing the microenvironment of mammary tumors using multiphoton microscopy. J Mammary Gland Biol Neoplasia 11:151-163

15. Wyckoff JB, Wang Y, Lin EY et al (2007) Direct visualization of macrophage-assisted tumor cell intravasation in mammary tumors. Cancer Res 67:2649-2656

16. Sahai E, Wyckoff J, Philippar U et al (2005) Simultaneous imaging of GFP, CFP and collagen in tumors in vivo using multiphoton microscopy. BMC Biotechnol 5:14

17. Seppen J, Barry SC, Klinkspoor JH et al (2000) Apical gene transfer into quiescent human and canine polarized intestinal epithelial cells by lentivirus vectors. J Virol 74:7642-7645

18. Neri A, Welch D, Kawaguchi T, Nicolson GL (1982) Development and biologic properties of malignant cell sublines and clones of a spontaneously metastasizing rat mammary adenocarcinoma. J Natl Cancer Inst 68:507-517

19. Huigsloot M, Tijdens IB, Mulder GJ, van de WB (2002) Differential regulation of doxorubicin-induced mitochondrial dysfunction and apoptosis by Bcl-2 in mammary adenocarcinoma (MTLn3) cells. J Biol Chem 277:35869-35879

20. Xue C, Wyckoff J, Liang F et al (2006) Epidermal growth factor receptor overexpression results in increased tumor cell motility in vivo coordinately with enhanced intravasation and metastasis. Cancer Res 66:192-197

21. Le Devedec SE, van Roosmalen W, Maria N et al (2009) An improved model to study tumor cell autonomous metastasis programs using MTLn3 cells and the Rag2(-/-) gammac $(-/-)$ mouse. Clin Exp Metastasis

22. Wyckoff J, Wang W, Lin EY et al (2004) A paracrine loop between tumor cells and macrophages is required for tumor cell migration in mammary tumors. Cancer Res 64:7022-7029

23. Zoumi A, Yeh A, Tromberg BJ (2002) Imaging cells and extracellular matrix in vivo by using second-harmonic generation and two-photon excited fluorescence. Proc Natl Acad Sci U S A 99:11014-11019

24. Campagnola PJ, Millard AC, Terasaki M et al (2002) Threedimensional high-resolution second-harmonic generation imaging of endogenous structural proteins in biological tissues. Biophys $\mathrm{J}$ 82: 493-508
25. Campagnola PJ, Loew LM (2003) Second-harmonic imaging microscopy for visualizing biomolecular arrays in cells, tissues and organisms. Nat Biotechnol 21:1356-1360

26. Shin KJ, Wall EA, Zavzavadjian JR et al (2006) A single lentiviral vector platform for microRNA-based conditional RNA interference and coordinated transgene expression. Proc Natl Acad Sci U S A 103:13759-13764

27. Amoh Y, Bouvet M, Li L et al (2006) Visualization of nascent tumor angiogenesis in lung and liver metastasis by differential dual-color fluorescence imaging in nestin-linked-GFP mice. Clin Exp Metastasis $23: 315-322$

28. Hoffman RM, Yang M (2005) Dual-color, whole-body imaging in mice. Nat Biotechnol 23:790

29. Hoffman RM, Yang M (2006) Whole-body imaging with fluorescent proteins. Nat Protoc 1:1429-1438

30. Yamamoto N, Yang M, Jiang P et al (2003) Determination of clonality of metastasis by cell-specific color-coded fluorescent-protein imaging. Cancer Res 63:7785-7790

31. Yang M, Baranov E, Jiang P et al (2000) Whole-body optical imaging of green fluorescent protein-expressing tumors and metastases. Proc Natl Acad Sci U S A 97:1206-1211

32. Yang M, Baranov E, Li XM et al (2001) Whole-body and intravital optical imaging of angiogenesis in orthotopically implanted tumors. Proc Natl Acad Sci U S A 98:2616-2621

33. Yang M, Li L, Jiang P et al (2003) Dual-color fluorescence imaging distinguishes tumor cells from induced host angiogenic vessels and stromal cells. Proc Natl Acad Sci U S A 100:14259-14262

34. Yang M, Jiang P, Yamamoto N et al (2005) Real-time whole-body imaging of an orthotopic metastatic prostate cancer model expressing red fluorescent protein. Prostate 62:374-379

35. Yang M, Jiang P, Hoffman RM (2007) Whole-body subcellular multicolor imaging of tumor-host interaction and drug response in real time. Cancer Res 67:5195-5200

36. Medjkane S, Perez-Sanchez C, Gaggioli C, Sahai E, Treisman R (2009) Myocardin-related transcription factors and SRF are required for cytoskeletal dynamics and experimental metastasis. Nat Cell Biol $11: 257-268$

37. Giampieri S, Manning C, Hooper S et al (2009) Localized and reversible TGFbeta signalling switches breast cancer cells from cohesive to single cell motility. Nat Cell Biol 11:1287-1296

38. Shaner NC, Patterson GH, Davidson MW (2007) Advances in fluorescent protein technology. J Cell Sci 120:4247-4260

39. Winnard PT Jr, Kluth JB, Raman V (2006) Noninvasive optical tracking of red fluorescent protein-expressing cancer cells in a model of metastatic breast cancer. Neoplasia 8:796-806

40. Kawano H, Kogure T, Abe Y, Mizuno H, Miyawaki A (2008) Twophoton dual-color imaging using fluorescent proteins. Nat Methods 5:373-374

41. Kedrin D, Gligorijevic B, Wyckoff J et al (2008) Intravital imaging of metastatic behavior through a mammary imaging window. Nat Methods 5:1019-1021 